Martin Bichler

\title{
Aufbau unternehmensweiter WWW-Informationssysteme
}




\section{Multimedia Engineering hrsg. von Wolfgang Effelsberg und Ralf Steinmetz}

Ziel der Reihe ist es, den Leser über Grundlagen und Anwendungen der Multimedia-Technik und der Telekooperation zu informieren. Die Reihe umfaßt Lehrbücher, einführende und umfassende Standardwerke sowie speziellere Monographien zu den Themen Multimedia, Hypermedia und computergestütze Kooperation. Es geht dabei beispielsweise um Fragen aus den Bereichen Betriebssysteme, Rechnernetze Kompressionsverfahren und grafische Oberflächen. In der Art der Darstellung wendet sie sich an Informatiker und Ingenieure, an Wissenschaffler, Studenten und Praktiker, die sich über dieses fazinierende und interdisziplinäre Thema informieren wollen.

Bisher erschienen:

Synchronisation in kooperativen Systemen

von Erwin Mayer

Multimediale Kiosksysteme

von Wieland Holfelder

Bildkompression mit Fraktalen

von Michael F. Barnsley und Lyman P. Hurd

Multimedia, Hypertext und Internet

von Jakob Nielsen

Entwicklung verteilter Multimedia-Applikationen

von Thomas Käppner

Aufbau unternehmensweiter WWW-Informationssysteme

von Martin Bichler

\section{Vieweg}


Martin Bichler

\section{Aufbau \\ unfernehmensweiter \\ WWW-Informationssysteme}

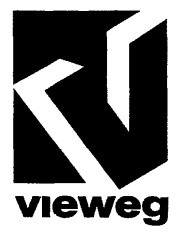


Alle Rechte vorbehalten

(C) Friedr. Vieweg \& Sohn Verlagsgesellschaft mbH, Braunschweig/Wiesbaden, 1997

Softcover reprint of the hardcover 1st edition 1997

Der Verlag Vieweg ist ein Unternehmen der Bertelsmann Fachinformation GmbH.

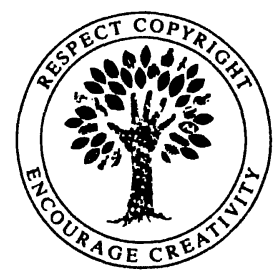

Das Werk einschließlich aller seiner Teile ist urheberrechtlich geschützt. Jede Verwertung außerhalb der engen Grenzen des Urheberrechtsgesetzes ist ohne Zustimmung des Verlags unzulässig und strafbar. Das gilt insbesondere für Vervielfältigungen, Übersetzungen, Mikroverfilmungen und die Einspeicherung und Verarbeitung in elektronischen Systemen.

http://www.vieweg.de

Druck und buchbinderische Verarbeitung: Lengericher Handelsdruckerei, Lengerich Gedruckt auf säurefreiem Papier 


\section{Vorwort}

"Although information presentation is, at the moment, the most important Web application this will change rapidly over the next year or two. "[siehe Maur96, 36]

Die Aufgabe des Informationsmanagements ist es, eine Informationsinfrastruktur bereitzustellen, die die Erfolgspotentiale des Betriebes sichert und weiter ausbaut. Die Mitarbeiter sollten möglichst umfassend mit Information versorgt werden. Im Laufe der Zeit wurden die verschiedensten Techniken angewendet, um dieses Ziel zu erreichen. Die Systeme wu rden immer mächtiger, die Benutzer konnten aber nicht im gleichen Ausmaß davon profitieren. Verschiedene Betriebssysteme, verschiedene Datenformate und unterschiedliche Benutzeroberflächen führten zu einer fast „babylonischen Sprachverwirrung" und verlangen vom Benutzer erhebliches EDV-technisches Fachwissen, um daraus die für ihn wichtige Information zu extrahieren. Das World Wide Web (WWW, Web) ist unabhängig vom Ort und der verwendeten Plattform einfach zu bedienen. Dokumente, die über das WWW zur Verfügung gestellt werden, können von jedem Rechner im Internet aus abgerufen werden. Zahlreiche Experten versprechen, daß dadurch viele Bereiche der betrieblichen Informationsverarbeitung revolutioniert werden. Diese Arbeit beschreibt den Aufbau unternehmensweiter Informationssysteme auf Basis des World Wide Web. Welches Potential diese Technik für das Informationsmanagement hat, läßt sich am besten durch einen kurzen Rückblick auf die Entwicklungsgeschichte betrieblicher Informationsverarbeitung zeigen.

Viele der Altsysteme basier(t)en auf proprietären Hostlösungen. Diese boten eine homogene, zentralisierte Umgebung, die von wenigen Produktspezialisten administriert wurde. 
Von Client-

Server-Systemen

Man begab sich aber dadurch auch in die Abhängigkeit der großen Anbieter und war durch die hohen Investitionskosten über Jahre hinaus an diese gebunden. Zudem war es sehr schwer, diese starren Systeme dem Firmenwachstum anzupassen. „Die Notwendigkeit zur Flexibilität und die Konzentration auf das Kerngeschäft führen dazu, daß sich große, monolithische Unternehmen zunehmend zu Netzwerken kleiner, flexibler und selbstverantwortlicher Geschäftseinheiten wandeln" [siehe ÖsRi96, 138]. Eine der größten Neuerungen in der betrieblichen Informationsverarbeitung der letzten Jahre waren daher Client-Server-Architekturen. Unter ClientServer-Architekturen versteht man eine kooperative Datenverarbeitung, bei der verschiedene Aufgaben unter verbundenen Rechnern aufgeteilt werden [vgl. Hans96c, 64].

Durch den Einzug von PCs in die Unternehmen konnten die bis dato "dummen" Terminals durch relativ "intelligente" Arbeitsplatzrechner ersetzt werden, die selbst Aufgaben wie Textverarbeitung oder Datenaufbereitung übernahmen. Mit der fortschreitenden Vernetzung entstand eine Art Arbeitsteilung zwischen PCs, UNIX-Workstations und Großrechnern. Diese Arbeitsteilung soll eine Reihe von Zwecken verfolgen. Hardware- wie auch Softwareressourcen sollen von den Mitarbeitern gemeinsam genutzt und dadurch besser ausgelastet werden. Ein weiteres wichtiges Argument ist Skalierbarkeit. Dieses als Downsizing, Upsizing beziehungsweise Rightsizing bezeichnete Merkmal bringt es mit sich, daß die IT-Infrastruktur relativ schnell an die betrieblichen Erfordernisse angepaßt werden kann. Im Laufe der Zeit entstanden die verschiedensten Arten von Client-Server-Systemen, von einfachen Datei-Servern bis zu Transaktionsservern und verteilten Objekten. Abbildung 1 veranschaulicht das zeitliche Aufkommen verschiedener Client-Server-Techniken. 
Abbildung 1:

Client-Server-

Entwicklung [nach OrHa97, 54] zu netzwerkzentrierten, WWWbasierten Informationssystemen

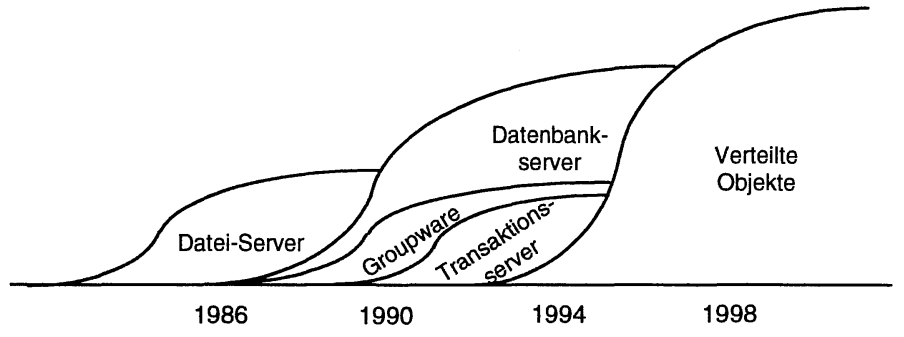

All diese Systeme stammen von verschiedenen Herstellern, haben unterschiedliche Betriebssysteme, unterschiedliche Benutzeroberflächen, unterschiedliche Formate und können daher nur schwer miteinander kommunizieren. Neben einem beachtlichen Know-how, das vom Benutzer verlangt wird, ist vor allem die Administration solch heterogener Systemlandschaften ein äußerst schwieriges Unterfangen. Client-ServerEntwicklung hat mittlerweile den Ruf, teuer und kompliziert zu sein. Verantwortlich dafür ist das Konglomerat aus proprietären Entwicklungswerkzeugen, Schnittstellen, Plattformen und Netzwerkprotokollen.

Das World Wide Web hat daneben seit 1989 eine fast revolutionäre Entwicklung durchgemacht und bietet nun ganz neue Optionen für betriebliche Anwendungen. Durch den Einsatz von Internetdiensten in immer mehr Bereichen etabliert sich der WWW-Browser als universeller Client. Der Benutzer hat es für verschiedene Arbeitsabläufe immer mit der gleichen Benutzeroberfläche zu tun, was die Bedienung von Programmen vereinfacht und Fehlerquellen reduziert. Entwicklungen wie Suns Java erhöhen auch die Funktionalität der Browser beträchtlich. "Client/server's future is on the Web " eine Schlagzeile aus der Computerworld [siehe Groc95, 18] verdeutlicht die Erwartungen der Softwarehersteller. Grund dafür sind eine Reihe bestechender Vorteile:

Das WWW ist plattformunabhängig. In heterogenen Systemen entfallen teure Softwareportierungen beziehungsweise die Cross-Plattformentwicklung. Große Vorteile bietet die Verwendung verbreiteter Internet-Standards wie TCP/IP, der 
Hypertext Markup Language (HTML), des Hypertext Transfer Protocol (HTTP) oder des Simple Mail Transfer Protocol (SMTP). Sie haben sich in zahlreichen großen Installationen bewährt und sind nicht proprietär. Außerdem ist damit die Anbindung von Intranets an das Internet denkbar einfach und erlaubt es externen und mobilen Benutzern auf Unternehmensdaten zuzugreifen. Darüber hinaus macht die Standardisierung den unkomplizierten Informationsaustausch zwischen innerbetrieblichen Informationssystemen und Geschäftspartnern möglich [vgl. Somm96, 9].

$\Rightarrow$ Sichere Protokolle wie Netscapes SSL, PCT oder S-HTTP erlauben, daß betriebliche Anwendungen auch im Web eingesetzt werden können. Das ist vorteilhaft für Unternehmen mit ausgeprägter Filialstruktur (Handel, Banken), für Elektronischen Handel oder für Tele-Working. Mit dem Web-Browser als Benutzerclient können von überall aus Anwendungen gestartet werden. Dadurch entfällt auch das leidige Problem der Verteilung neuer Software-Releases.

$\Rightarrow$ Mit den Hypermedia-Fähigkeiten und den Elementen klassischer grafischer Benutzeroberflächen kann der Entwickler ergonomische Anwendungen erstellen.

$\Rightarrow$ Web-Techniken ermöglichen es, operative Altsysteme mit einer neuen Benutzeroberfläche auszustatten. Relativ kostengünstig lassen sich WWW-Clients erstellen. Zumindest für die gängigen Datenbanken gibt es brauchbare Ansätze.

$\Rightarrow \quad$ E-Mail und elektronische Diskussionslisten können, richtig eingesetzt, die innerbetriebliche Kommunikation und Organisation verbessern. Die Bausteine einer IntranetLösung sind zum Großteil gratis im Netz zu beziehen.

Bis zur Jahrtausendwende sollen über zwei Drittel der deutschen Unternehmen auf Intranets zugreifen können [vgl. Comp97]. Zona Research prophezeit ein Umsatzwachstum um das 16fache für Intranet-Software auf acht Milliarden Dollar innerhalb der nächsten drei Jahre [vgl. Somm96, 9]. Fast alle 
großen Softwareunternehmen sind daher bemüht, ihre Produkte WWW-tauglich zu machen beziehungsweise mit WWW-Oberflächen auszustatten [vgl. auch Born96]. Das Web ist auf dem Weg, zur universellen Benutzerschnittstelle zu den verschiedensten betrieblichen IS-Ressourcen zu werden von Textdokumenten über Datenbanken, Groupware bis hin zu integrierter betriebswirtschaftlicher Standardsoftware wie SAP. „Das Internet entwickelt sich zum bedeutendsten Integrator der Informatikgeschichte. Standards wie HTTP, FTP und SMTP sowie das universelle Adressierungssystem URL haben vielfältige Applikationen und Plattformen kommunikationsfähig gemacht“" [siehe ÖsRi96, 19].

Zielsetzung Vor diesem Hintergrund ist dieses Buch entstanden. Es stellt den State-of-the-Art WWW-basierter Informationssysteme dar, sowohl hinsichtlich der technischen Grundlagen als auch der praktischen Umsetzung von WWW-Projekten. Es soll helfen,

$\Rightarrow$ sich im komplexen Themengebieten unternehmensweiter WWW-Informationssysteme zurecht zu finden und dabei Techniken wie HTML, Java, CORBA oder Datenbankintegration einordnen zu können.

$\Rightarrow$ die Komponenten unternehmensweiter WWW-Informationssysteme sowie den Aufbau der nötigen IT-Infrastruktur $\mathrm{zu}$ identifizieren und

$\Rightarrow$ Methoden und Vorgehenskonzept für die Umsetzung konkreter WWW-Projekte zu finden.

Das Buch wendet sich an Softwareentwickler mit objektorientierten Kenntnissen, Leiter und Mitarbeiter von WWW-Projekten, Informationssystem-Manager sowie Studenten und Dozenten der Informatik und Wirtschaftsinformatik.

Danksagung Ohne die Unterstützung vieler Freunde hätte dieses Buch nicht entstehen können. Besonderer Dank gilt Herrn Professor Hans Robert Hansen für die Förderung der Arbeit, seine Unterstützung und die hervorragenden Rahmenbedingungen am Institut für Wirtschaftsinformatik der Wirtschaftsuniversität Wien. Zu größtem Dank bin ich meinem Freund und Kollegen Dr. Stefan Nusser verpflichtet, mit dem auch gemeinsam 
die WWW-Entwurfsmethode W3DT entwickelt wurde. Vor allem Claudia Stoiss und auch andere Freunde aus meinem privaten Umfeld halfen mir mit aufmunternden Worten über gelegentliche Durststrecken hinweg. Dr. Christian Bauer, Dipl.-Ing. Willi Langenberger, Dr. Andreas Wildberger sowie allen anderen Kolleginnen und Kollegen an der Abteilung für Wirtschaftsinformatik danke ich für zahlreiche Anregungen und Gespräche.

Wien, im Juli 1997

der Autor 


\section{Inhaltsverzeichnis}

1 Einleitung ............................................................................ 1

1.1 Unternehmensweite WWW-Informationssysteme .......... 1

1.2 Zielsetzung des Buches ............................................... 4

2 Grundlegende www-Techniken.................................... 8

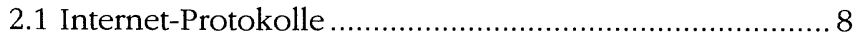

2.2 Architektur und Funktionalität des WWW .................. 13

2.3 Hypermedia und HTML ............................................. 19

2.4 Web-Server und Clients ............................................. 21

2.4.1 Erzeugen dynamischer Dokumente 23

2.4.2 Einfache Arten der Zugriffskontrolle 24

2.4.3 Sicherung von Teilnetzen durch Firewalls $\quad 26$

2.5 Konzeptionelle Probleme ........................................... 28

2.6 Exkurs: Hyperwave ................................................. 31

3 IT-Infrastruktur unternehmensweiter Www-

Informationssysteme ......................................................... 33

3.1 Aufbau der IT-Infrastruktur ........................................ 33

3.1.1 IT-Infrastruktur und IS-Architektur $\quad 33$

3.1.2 WWW als Basis unternehmensweiter Informationssysteme 35

3.1.3 Vier-Schichtenmodell 39

3.2 Präsentationsschicht ...................................................... 41

3.2.1 Java 42

3.2.2 Remote Programming mit Agenten 48

3.3 Dienst- und Vermittlungsschicht ............................... 54

3.3.1 Transaktionssicherheit im WWW 56

3.3.2 Abrechnungssysteme 64

3.3.3 Such- und Retrievalinstrumente $\quad 67$ 
3.3.4 Benutzer- und Ressourcenverwaltung $\quad 70$

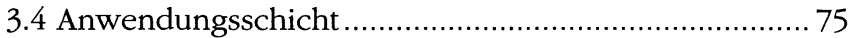

3.4.1 Probleme CGI-basierter WWW-Anwendungen 75

3.4.2 Einsatz von Mobile-Code-Systemen 80

3.4.3 WWW und objektorientierte Middleware $\quad 83$

4 Entwicklung von WWW-Informationssystemen .........94

4.1 Neue Anforderungen an das Informations-

management ............................................................ 97

4.2 Vorgehen bei der Entwicklung unternehmensweiter-

WWW-Informationssysteme.......................................98

4.2.1 Vergleich zur konventionellen

Softwareentwicklung 98

4.2.2 Entwicklung eines WWW-Rahmenkonzeptes 102

4.3 Anforderungsanalyse ............................................... 104

4.3.1 Unternehmensorganisation und betriebliche IT-

Infrastruktur 105

4.3.2 Zielgruppenanalyse $\quad 106$

4.3.3 Anforderungsspezifikation $\quad 107$

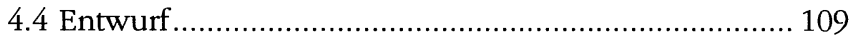

4.4.1 Informationsstrukturierung und

Navigationsentwurf 112

4.4.2 Organisations- und Oberflächenentwurf 124

4.4.3 Computergestützter Entwurf von WWW-

Informationssystemen $\quad 126$

4.4.4 Beispiel VideoWeb 128

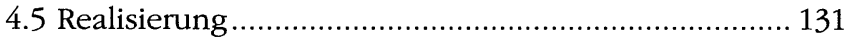

4.5.1 Netscape Merchant System 132

4.5.2 SAP R/3 Internet Application Components 135

4.5.3 Eigenentwicklung mit Oracle CASE-

Werkzeugen

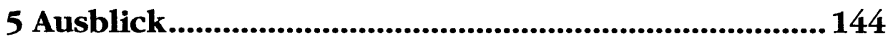

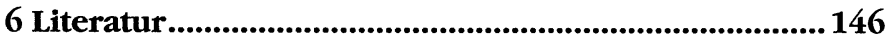


7 Glossar.

8 Abkürzungsverzeichnis 166

9 Sachwortverzeichnis 170 